Special issue of the International Conference on Computational and Experimental Science and Engineering (ICCESEN 2014)

\title{
Semi-Empirical Formula with New Coefficients of the $(\alpha, n)$ Reaction Cross-Section
}

\author{
S. AKÇA ${ }^{a, *}$, E. TEL $^{b}$ AND S. GÜNGÖR ${ }^{a}$ \\ ${ }^{a}$ Çukurova University, Faculty of Arts and Science, Department of Physics, Adana, Turkey \\ ${ }^{b}$ Osmaniye Korkut Ata University, Faculty of Arts and Science, Department of Physics, Osmaniye, Turkey

\begin{abstract}
A new modified semi-empirical formula including non-elastic scattering and Coulomb effects for the $(\alpha, \mathrm{n})$ reaction cross-section was obtained. The available experimental data were used to get the $(\alpha, \mathrm{n})$ reaction cross-section systematics. The dependence between the cross-section and asymmetry term was discussed for $(\alpha, \mathrm{n})$ reaction at $18.5 \pm 3 \mathrm{MeV}$ energies classifying the target nuclei into odd $Z$-even $N$ and even $Z$-even $N$. The coefficients of determination $\left(R^{2}\right)$ for all classifications were detected to exhibit the amount of relation between asymmetry term and cross-sections obtained from the modified semi-empirical formula with new coefficients.
\end{abstract}

DOI: 10.12693/APhysPolA.128.B-128

PACS: 25.40.-h, 25.60.Dz

\section{Introduction}

When experimental data are limited or to determine unmeasured data, generally, the systematics of nuclear reaction cross-sections is needed. Therefore, knowing the systematics of reaction cross-sections is of great importance.

A great number of empirical and semi-empirical crosssection formulae have been proposed for different target nuclei irradiated with uncharged neutron and charged particle at the specific energies [1-12]; however, there is not any study on cross-section systematics of alphainduced reactions. As a result of alpha-induced reactions, in particular the production of significant medical radioisotopes used in nuclear medicine occurs [13-15].

The aim of this study is to develop a semi-empirical formula for odd $Z$-even $N$ and even $Z$-even $N$ target nuclei of the $(\alpha, \mathrm{n})$ reaction cross-sections at $18.5 \pm 3 \mathrm{MeV}$ alpha energies. The target mass regions which are investigated in this paper are between $41 \leq A \leq 209$. The formulae are based on non-elastic effects of optical model and the binding energy systematics of the nuclear shell model, with dependence on the pairing and Coulomb effects. The expressions in the shell model were adapted to the mechanism of nuclear reactions.

\section{Basic systematics and the models used in the existing formulae}

There are many studies on systematics of the crosssection in the literature. Several empirical and semiempirical cross-section formulae have been proposed by the authors for both neutron and also charged particle induced reactions. First, Levkovskii showed that $(n, p)$ cross-sections of many isotopes of one element decrease regularly with increasing $A$ for $14 \mathrm{MeV}$ neutrons.

\footnotetext{
*corresponding author; e-mail: sblakca@gmail.com
}

The cross-section is expressed with an exponential function depending on $Z$ and $A$ of the target in experimental approach. The character of variation of empirical $(n, p)$ cross-sections at $14 \mathrm{MeV}$ was presented as

$$
\begin{gathered}
\alpha_{\mathrm{p}}=\frac{\sigma(A+\Delta A, Z)}{\sigma(A, Z)} \approx \exp \left(75\left(\frac{Z}{A+\Delta A}-\frac{Z}{A}\right)\right) \approx \\
\quad \exp \left(-33 \frac{\Delta A}{A}\right) .
\end{gathered}
$$

This formula can be considered as a reflection of a general theory relating the probability of emission of a proton from the struck nucleus with a proton "concentration" $Z / A$.

When analyzed the experimental data by Levkovskii, the cross-sections were described as

$$
\sigma_{\mathrm{n}, \mathrm{p}}=\sigma_{\mathrm{c}}(n) \alpha_{\mathrm{p}}
$$

where $\sigma_{\mathrm{c}}(n)$ is the geometrical cross-section of the nucleus, and given as $\pi r_{0}^{2}\left(A^{1 / 3}+1\right)^{2}[\mathrm{mb}]$. The $\sigma_{\mathrm{n}, \mathrm{p}}$ empirical formula in Eq. (2) of Levkovskii turned into Eq. (3) [1]:

$$
\sigma_{\mathrm{n}, \mathrm{p}}(\mathrm{mb})=45.2\left(A^{1 / 3}+1\right)^{2} \exp \left(-33 \frac{N-Z}{A}\right) .
$$

$(\mathrm{n}, \mathrm{p})$ cross-section formula at $14 \mathrm{MeV}$ proposed by Levkovskii reveals the exponential dependence of crosssection on asymmetry term $(s=N-Z / A)$.

Most authors have provided theoretical support to Levkovskii's experimental formula for $(\mathrm{n}, \mathrm{x})$ reactions. Pai et al. added an effective $Q$-value into the statistical model calculation for $(\mathrm{n}, \mathrm{p})$ reactions at $14 \mathrm{MeV}$ [2]. Belgaid and Asghar derived a semi-empirical $(\mathrm{n}, \alpha)$ cross-section formula at $14.5 \mathrm{MeV}$ based on the evaporation model. In the formula, droplet model of Myers and Swiatecki [16] was used for $Q(\mathrm{n}, \alpha)$ calculations [4]. Habbani and Osman proposed systematics for $(\mathrm{n}, \mathrm{p}),(\mathrm{n}, \alpha)$, and $(\mathrm{n}, \mathrm{np})$ reaction cross-section for $14.5 \mathrm{MeV}$ neutrons and the obtained formula was based on the statistical model with the $Q$-value and odd-even effect [6]. Tel et al. obtained a new empirical $(\mathrm{n}, \mathrm{p})$ cross-section formula tak- 
ing shell model and pairing effect into consideration by modifying Levkovskii's empirical formula [7]. Broeders and Konobeyev proposed semi-empirical formula of $(n, p)$ reaction cross-section at $14.5,20,30 \mathrm{MeV}$ neutron energies. The formula takes into account the contribution of both non-equilibrium proton emission and also equilibrium proton emission [8].

The studies on systematics of $(\mathrm{p}, \mathrm{x})$ reaction crosssections were carried out by some authors. For example, Broeders and Konobeyev obtained semi-empirical systematics of $(\mathrm{p}, \alpha)(\mathrm{p}, \mathrm{n} \alpha)$, and $(\mathrm{p}, \mathrm{np})$ reaction crosssections at various incident proton energies from 17.9 to $28.5 \mathrm{MeV}$ and $(\mathrm{p}, \mathrm{n})$ reaction cross-section at incident proton energies $7.5,12.4,24.8 \mathrm{MeV}$. The systematics were based on analytical formulae derived using the preequilibrium exciton model, evaporation model and semiempirical mass formula $[9,10]$. Then, Tel et al. suggested semi-empirical formula that includes non-elastic effects of optical model for $(\mathrm{p}, \alpha)$ at $17.9 \mathrm{MeV}$ and $(\mathrm{p}, \mathrm{np})$ at 22.3 MeV. Besides, they investigated Coulomb and pairing effects for $(\mathrm{p}, \alpha)$ at $17.9 \mathrm{MeV},(\mathrm{p}, \mathrm{np})$ at $22.3 \mathrm{MeV}$, $(\mathrm{p}, \mathrm{n} \alpha)$ at 24.8 and $28.5 \mathrm{MeV}$ and presented a new formula. That novel formula was derived by taking advantage of Levkovskii's original formula with asymmetry term $[11,12]$.

Up to now in the literature, there has not been any systematic study on reaction cross-section of the alpha induced reactions. The $(\alpha, \mathrm{n})$ reaction cross-section formulae proposed in the present work are based on nonelastic effect of optical model and Coulomb effect in the binding energy of the nuclear shell model.

\section{Results and discussion}

Dependence upon asymmetry term of reaction crosssection has been investigated for several particle induced reactions $[1-3,7,11,12]$. The exponential dependence of $(\alpha, \mathrm{n})$ reaction cross-section on asymmetry parameter can be given as follows:

$$
\sigma(\alpha, n)=B \exp (a(N-Z) / A),
$$

where $\sigma(\alpha, n)$ is the $(\alpha, n)$ cross-section, value $(N-$ $Z) / A=s$ is asymmetry term and $B, a$ are the coefficients. Actually, here $\sigma(\alpha, n)$ represents maximum crosssection in the experimental data used. The coefficients $B$, $a$ and coefficient of determination $R^{2}$ belonging to the exponential dependence in Eq. (4) are given in Table I using available experimental data at $18.5 \pm 3 \mathrm{MeV}$. The dependence of above-mentioned empirical cross-section upon asymmetry parameter is shown in Figs. 1-3 for all nuclei, odd $Z$-even $N$ and even $Z$-even $N$ nuclei, respectively.

The coefficient of determination $\left(R^{2}\right)$ denotes the strength of the linear association between $\ln (\sigma(\alpha, \mathrm{n}))$ and the asymmetry term. As seen in Table I and Figs. 1-3, the values $R^{2}$ are quite big and the correlation between the measured $(\alpha, \mathrm{n})$ reaction cross-sections and asymmetry term for even $Z$-even $N$ nuclei is stronger than the correlation for odd $Z$-even $N$ and all nuclei.
TABLE I

Empirical and semi-empirical cross-section formulae and the new obtained coefficients for $(\alpha, \mathrm{n})$ reactions at $18.5 \pm 3 \mathrm{MeV}$ incident energies. $\sigma(\alpha, n)=B \exp (a s)$.

\begin{tabular}{c|c|c|c|c}
\hline \hline$Z$ & $N$ & $B$ & $a$ & $R^{2}$ \\
\hline \multicolumn{2}{c|}{ all nuclei } & 4820.45 & -21.861 & 0.836 \\
\cline { 1 - 1 } odd & even & 4104.72 & -21.006 & 0.795 \\
even & even & 5388.37 & -22.358 & 0.859
\end{tabular}

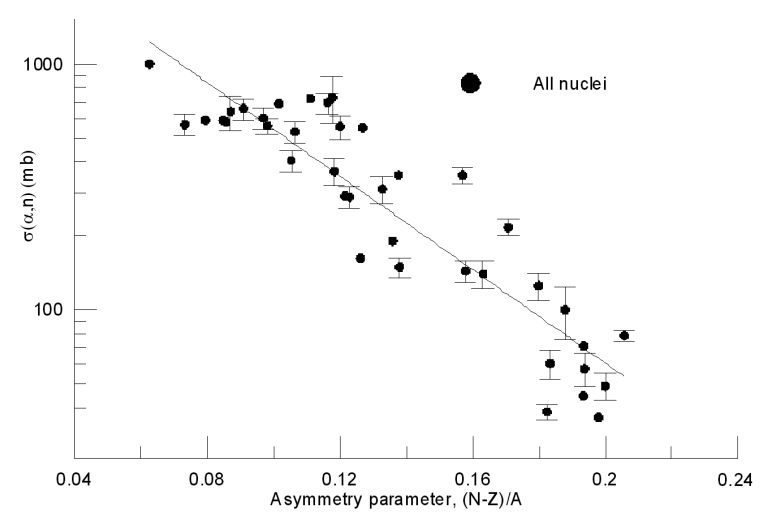

Fig. 1. The $(\alpha, \mathrm{n})$ reaction cross-sections depending upon asymmetry parameter for all nuclei at $18.5 \pm$ $3 \mathrm{MeV}$.

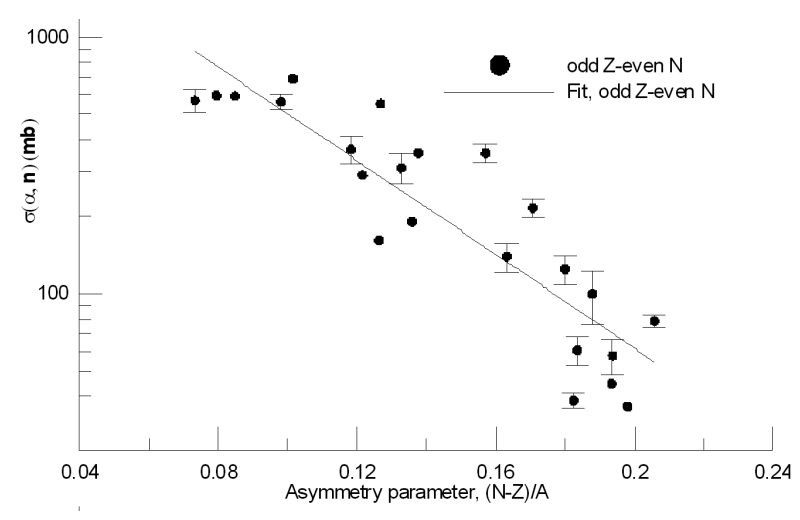

Fig. 2. As in Fig. 1, but for odd $Z$-even $N$ nuclei.

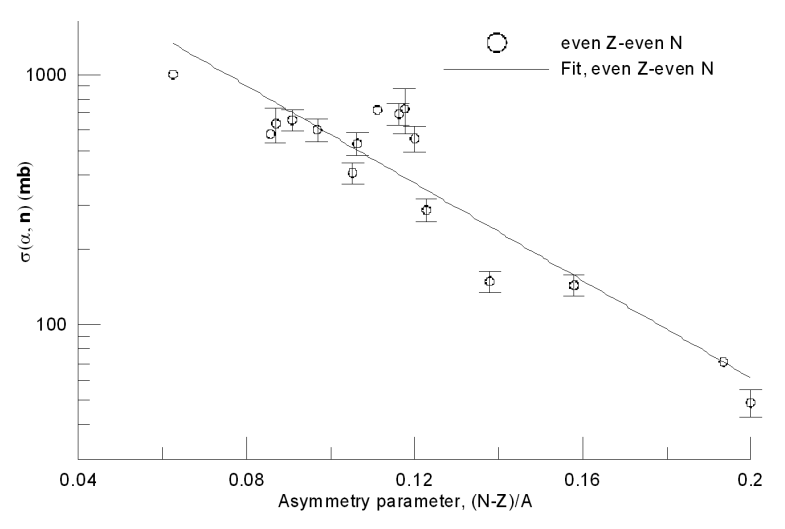

Fig. 3. As in Fig. 1, but for even $Z$-even $N$ nuclei. 
If the contribution of non-elastic scattering on the reaction cross-section is taken into account, the $(\alpha, \mathrm{n})$ reaction cross-section includes alpha non-elastic cross-section expression as below

$$
\sigma_{\mathrm{ne}}=\pi r_{0}^{2}\left(A^{1 / 3}+4^{1 / 3}\right)^{2} .
$$

$\sigma_{\text {ne }}$ is the alpha non-elastic scattering cross-section and this value also represents the geometrical cross-section of the reaction.

Alphas which are being charged particles affect every other protons in the nucleus when interacting with target nucleus and create a compound nucleus. The Coulomb potential for alpha particles is written as follows:

$$
\begin{aligned}
& V_{\text {Coul. }}=k \frac{(z+Z)(z+Z-1) e^{2}}{R} \approx k \frac{Z^{2} e^{2}}{R}, \quad z \ll Z, \\
& V_{\text {Coul. }} \approx b \frac{Z^{2}}{A^{1 / 3}+4^{1 / 3}},
\end{aligned}
$$

where $z$ and $Z$ represent atom numbers of alpha particle and target nucleus, respectively. The cross-section including Coulomb effect can be approximately written as follows:

$$
\sigma_{\text {Coul. }} \approx c \frac{Z^{2}}{A^{1 / 3}+4^{1 / 3}},
$$

where the term $b$ and $c$ are constant coefficients.

The $\sigma(\alpha, \mathrm{n})$ empirical cross-sections including alpha non-elastic scattering in Eq. (5) and Coulomb crosssections in Eq. (7) at $18.5 \pm 3 \mathrm{MeV}$ alpha incident energies can be given as follows [12]:

$$
\begin{aligned}
& \sigma(\alpha, \mathrm{n})=E \sigma_{\mathrm{ne}} \sigma_{\text {Coul. }} \exp (a s), \\
& \sigma(\alpha, \mathrm{n})=E^{\prime} Z^{2}\left(A^{1 / 3}+4^{1 / 3}\right) \exp (a s),
\end{aligned}
$$

where the coefficients $E, E^{\prime}$ and $a$ are determined using available empirical cross-sections taken from experimental data library. The values $E^{\prime}, a$ and coefficient of determination $R^{2}$ are obtained taking advantage of the exponential dependence in Eq. (9) and are given in Table II. The dependence of asymmetry parameter upon abovementioned empirical cross-section including non-elastic scattering and Coulomb effects is shown in Figs. 4-6 for all nuclei, odd $Z$-even $N$ and even $Z$-even $N$ nuclei.

TABLE II

Empirical and semi-empirical cross-section formulae and the new obtained coefficients including non-elastic scattering and Coulomb effects for $(\alpha, \mathrm{n})$ reactions at $18.5 \pm 3 \mathrm{MeV}$ incident energies. $\sigma(\alpha, \mathrm{n})=E^{\prime} Z^{2}\left(A^{1 / 3}+4^{1 / 3}\right) \exp (a s)$.

\begin{tabular}{c|c|c|c|c}
\hline \hline$Z$ & $N$ & $E^{\prime}$ & $a$ & $R^{2}$ \\
\hline \multicolumn{2}{c|}{ All nuclei } & 4.558 & -39.648 & 0.883 \\
\cline { 1 - 1 } odd & even & 7.072 & -42.745 & 0.900 \\
even & even & 2.293 & -33.757 & 0.830
\end{tabular}

According to Figs. 4-6, the linear dependence of $\ln \left(\sigma(\alpha, \mathrm{n}) / Z^{2}\left(A^{1 / 3}+4^{1 / 3}\right)\right)$ on $(N-Z) / A$ is very strong and the results of $R^{2}$ in Table II are $0.883,0.900$, and 0.830 for all nuclei, odd $Z$-even $N$, and even $Z$-even $N$ nuclei, respectively. When the non-elastic and Coulomb effects are included, the value $R^{2}$ for even $Z$-even $N$ nuclei is seen to be lower than the others.

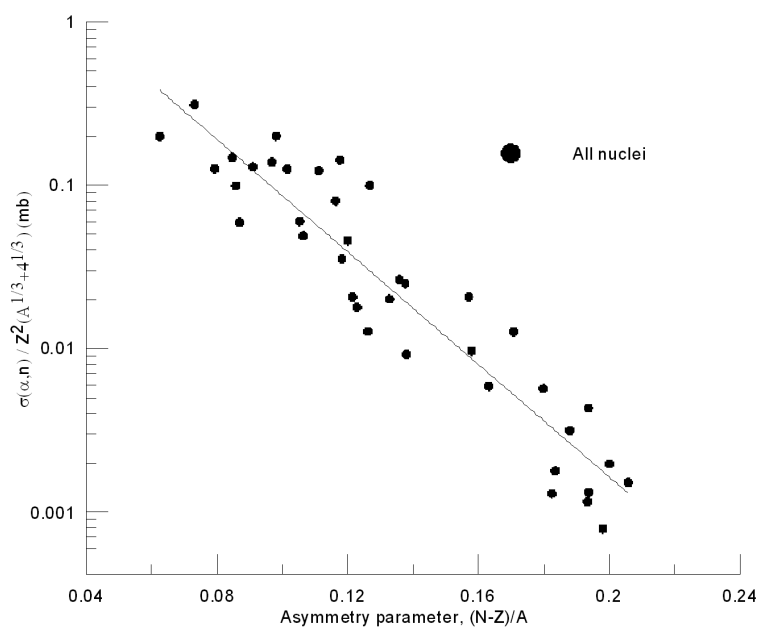

Fig. 4. The $(\alpha, \mathrm{n})$ reaction cross-sections including both non-elastic scattering and also Coulomb crosssection for all nuclei at $18.5 \pm 3 \mathrm{MeV}$ depending upon asymmetry parameter.

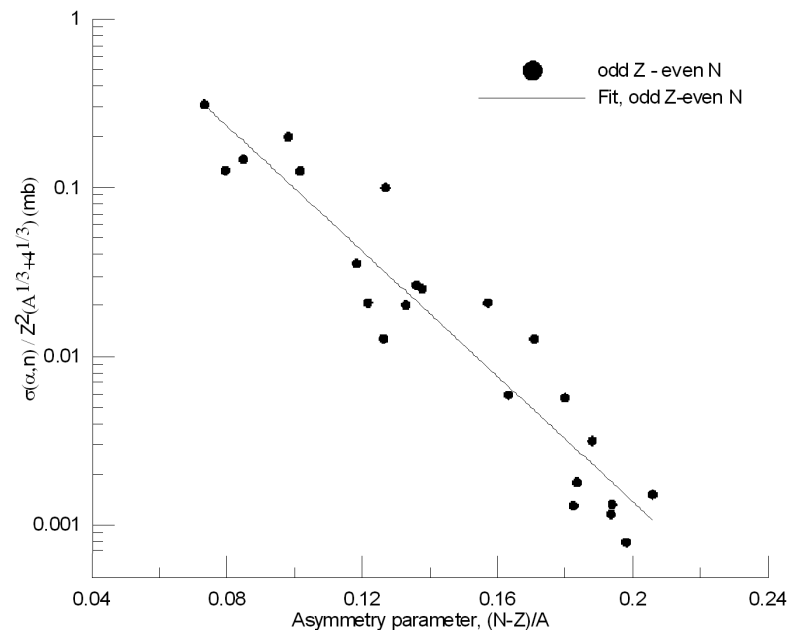

Fig. 5. As in Fig. 4 for odd $Z$-even $N$ nuclei.

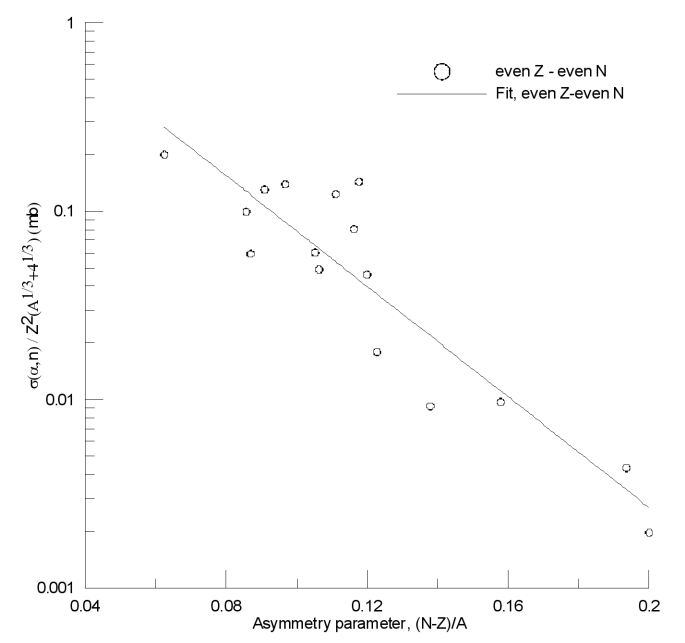

Fig. 6. As in Fig. 4 for even $Z$-even $N$ nuclei. 


\section{Conclusion}

A new semi-empirical formula including non-elastic and Coulomb cross-sections for the $(\alpha, \mathrm{n})$ reaction crosssection at $18.5 \pm 3 \mathrm{MeV}$ energies was suggested. The available experimental data of 38 target masses in the range of 41-209 were used. The non-elastic expression of the optical model and Coulomb expression of the nuclear shell model were used to get the new semi-empirical formula.

The relationship between available experimental data of $(\alpha, \mathrm{n})$ reaction cross-sections and asymmetry parameter was given for all, odd $Z$-even $N$ and even $Z$-even $N$ nuclei in Table I. The values $R^{2}$ greater than 0.7 indicate how much strong correlation is between $\ln (\sigma(\alpha, \mathrm{n}))$ and $(N-Z) / A$, and it is the strongest for even $Z$-even $N$ nuclei. As seen in Table II, the dependence between the $(\alpha, \mathrm{n})$ reaction cross-sections including non-elastic scattering and Coulomb effects and asymmetry term is quite strong $\left(R^{2} \geq 0.80\right)$. When the classification is made according to even $Z$-even $N$ and odd $Z$-even $N$ nuclei, the value $R^{2}$ for even $Z$-even $N$ nuclei is smaller. According to the results of tables, it is seen that the correlation of the $(\alpha, \mathrm{n})$ cross-sections including alpha non-elastic crosssection and Coulomb effect with asymmetry term are stronger than that of the measured experimental $(\alpha, \mathrm{n})$ cross-section data.

The semi-empirical formulae with new coefficient of $(\alpha, \mathrm{n})$ reaction cross-section obtained using non-elastic cross-section and Coulomb effect could be applied to provide a useful tool for estimating the cross-section with good accuracy.

\section{Acknowledgments}

This work was supported by Research Fund of Çukurova University, Project No. MMF2013D5 (364ID).

\section{References}

[1] V.N. Levkovskii, Sov. Phys. JETP 18, 213 (1964).

[2] H.L. Pai, D.G. Andrews, Can. J. Phys. 56, 944 (1978).

[3] I. Kumabe, K. Fukuda, J. Nucl. Sci. Technol. 24, 839 (1987).

[4] M. Belgaid, M. Ashgar, Nucl. Instrum. Methods Phys. Res. B 149, 383 (1999).

[5] M. Belgaid, T. Segueni, F. Kadem, M. Asghar, Nucl. Instrum. Methods Phys. Res. B 201, 545 (2003).

[6] F.I. Habbani, K.T. Osman, Appl. Radiat. Isotop. 54, 283 (2001).

[7] E. Tel, B. Şarer, Ş. Okuducu, A. Aydın, G. Tanır, J. Phys. G Nucl. Part. Phys. 29, 2169 (2003).

[8] C.H.M. Broeders, A.Yu. Konobeyev, Nucl. Phys. A 780, 130 (2006).

[9] C.H.M. Broeders, A.Yu. Konobeyev, Appl. Radiat. Isotop. 6, 1249 (2007).

[10] C.H.M. Broeders, A.Yu. Konobeyev, Radiochim. Acta 96, 387 (2008).

[11] E. Tel, A. Aydın, E.G. Aydın, A. Kaplan, Ö. Yavaş, İ.A. Reyhancan, Ind. Acad. Sci. 74, 931 (2010a).

[12] E. Tel, E.G. Aydın, A. Aydın, A. Kaplan, M.H. Bölükdemir, Ş. Okuducu, Phys. At. Nuclei 73, 412 (2010b).

[13] EXFOR/CSISRS (Experimental Nuclear Reaction Data), Database Version of February 26, 2013, Brookhaven National Laboratory, National Nuclear Data Center, 2013. http://www.nndc.bnl.gov/exfor/exfor00.htm.

[14] F. Tarkanyi, A. Hermanne, B. Kiraly, S. Takacs, A.V. Ignatyuk, Appl. Radiat. Isotop. 68, 404 (2010).

[15] H. Fuladvand, M. Bakhtiari, M. Sadeghi, M. Amiri, J. Radioanal. Nucl. Chem. 298, 501 (2013).

[16] W.D. Myers, W.J. Swiatecki, Ann. Phys. 84, 186 (1974). 